\title{
Correlation of PIK3CA mutation with programmed death ligand-1 (PD-L1) expression and their clinicopathological significance in colorectal cancer
}

\author{
Ae Ri Ahn ${ }^{1}$, Kyoung Min Kim ${ }^{1}$, Kyu Yun Jang ${ }^{1}$, Woo Sung Moon ${ }^{1}$, Gi Won $\mathrm{Ha}^{2}$, Min Ro Lee \\ Myoung Ja Chung ${ }^{1}$
}

${ }^{1}$ Departments of Pathology, Jeonbuk National University Medical School, Research Institute of Clinical Medicine of Jeonbuk National University, Biomedical Research Institute of Jeonbuk National University Hospital, and Research Institute for Endocrine Sciences, Jeonju, Republic of Korea; ${ }^{2}$ Department of Surgery, Jeonbuk National University Medical School, Research Institute of Clinical Medicine of Jeonbuk National University, Biomedical Research Institute of Jeonbuk National University Hospital, and Research Institute for Endocrine Sciences, Jeonju, Republic of Korea Contributions: (I) Conception and design: MJ Chung, AR Ahn, KM Kim, KY Jang, WS Moon; (II) Administrative support: AR Ahn, MJ Chung; (III) Provision of study materials or patients: AR Ahn, MJ Chung, MR Lee; (IV) Collection and assembly of data: AR Ahn, MR Lee, GW Ha, MJ Chung; (V) Data analysis and interpretation: AR Ahn, MJ Chung; (VI) Manuscript writing: All authors; (VII) Final approval of manuscript: All authors.

Correspondence to: Myoung Ja Chung, MD, PhD. Department of Pathology, Chonbuk National University Medical School, San 2-20 Keumam-Dong Dukjin-gu, Jeonju 54907, Republic of Korea. Email: mjchung@jbnu.ac.kr.

\begin{abstract}
Background: The prognostic significance of PIK3CA mutations in colorectal cancer (CRC) remains controversial. Recently, an association between programmed death ligand-1 (PD-L1) and PIK3CA mutations has been reported. The study presented here was conducted to investigate the effect of PIK3CA mutations on the prognosis of CRC patients and the association between PIK3CA mutations and PD-L1.

Methods: PIK3CA mutations were analyzed by targeted next-generation sequencing using formalinfixed paraffin-embedded specimens from 224 primary CRC patients. PD-L1 expression was evaluated by immunohistochemical staining.

Results: PIK3CA mutations and PD-L1 expression were detected in $21.4 \%$ and $10.3 \%$ of CRC patients, respectively. $P I K 3 C A$ mutations were significantly correlated with right-side colon cancer $(\mathrm{P}=0.011)$ and were correlated inversely with lymph node metastasis $(\mathrm{P}=0.026)$, distant metastasis $(\mathrm{P}=0.047)$, and high TNM stage $(\mathrm{P}=0.036)$. In univariate analysis, $P I K 3 C A$ mutations were correlated with longer relapse-free survival in CRC patients. PD-L1 expression was correlated significantly with $P I K 3 C A$ mutations $(\mathrm{P}<0.001)$.

Conclusions: PIK3CA mutations were associated with favorable prognostic factors, longer relapse-free survival, and expression of PD-L1. Further investigation is needed to identify whether PIK3CA mutations are a good prognostic factor. Additionally, further studies are needed to understand the mechanisms behind the correlation between PIK3CA mutations and PD-L1 expression.
\end{abstract}

Keywords: Colorectal cancer (CRC); PIK3CA; programmed death ligand-1 (PD-L1)

Submitted May 06, 2021. Accepted for publication Jul 25, 2021.

doi: 10.21037/atm-21-2315

View this article at: https://dx.doi.org/10.21037/atm-21-2315

\section{Introduction}

Colorectal cancer (CRC) is a common type of cancer. The number of new CRC cases worldwide has exceeded 1.8 million, and the number of deaths was estimated to be 881,000 in 2018 (1). Tumorigenesis and progression of CRC are multifactorial and dependent on lifestyle, the immune system, and genetic mutations. The main treatment for CRC is surgery and adjuvant chemotherapy. 
The relapse rate of CRC is approximately $30 \%$ (2). Due to the high relapse rate, recent studies have emphasized the importance of individually tailored treatments for better patient outcomes. The molecular characteristics of CRC provide important information for development of targeted therapies.

PIK3CA is a commonly mutated gene in CRC and encodes the p110 alpha (p110 $\alpha$ ) protein, which is a subunit of an enzyme called phosphatidylinositol 3-kinase (PI3K). PIK3CA mutations activates the $\mathrm{PI} 3 \mathrm{~K} /$ protein kinase $\mathrm{B}$ (PI3K-AKT) pathway and contribute to cellular growth, proliferation, and survival of the tumor cells (3). Early studies have suggested that PIK3CA mutations can influence tumor development and progression (4-6). PIK3CA mutations are thought to play an oncogenic role and act as a marker for poor prognosis and as a negative predictor for response to anti-epidermal growth factor receptor (EGFR) treatment in CRC (7). However, other reports have revealed that PIK3CA mutations are a favorable prognostic factor in CRC $(8,9)$, or that the prognostic relevance of PIK3CA mutations remains controversial $(10,11)$. Analysis of the cBioPortal database has revealed a significant correlation between PIK3CA mutations and prolonged disease-free survival in patients with CRC (12). PIK3CA mutations are observed in $24.7 \%$ (147 of 594) of CRC cases in The Cancer Genome Atlas (TCGA). Survival analysis of cases from the TCGA database disclosed no prognostic value for PIK3CA mutations in CRC (13).

Programmed death ligand-1 (PD-L1), a ligand for programmed death receptor-1 (PD-1), is expressed in tumor infiltrating immune cells and tumor cells. PD-1 is an inhibitory receptor expressed on activated T-cells. When PD-L1 expressed on tumor cells binds to its receptor, PD1 , it suppresses $\mathrm{T}$ cell function, downregulating the immune response. Targeting the PD-1/PD-L1 blockade has been proposed as a promising breakthrough in cancer treatment including that of CRC.

Previous studies have shown that genetic alterations within tumors can influence the engagement of the immune system. EGFR mutations or EML4-ALK fusions activate the PD-1/PD-L1 pathway via PD-L1 upregulation. PIK3CA mutations were associated with PD-L1 overexpression in gastric cancer (14). In this study, we analyzed PIK3CA mutations and PD-L1 expression in 224 CRC specimens and examined the correlation between the two. We also investigated their correlation with clinicopathologic features of CRC. We present the following article in accordance with the REMARK reporting checklist (available at https:// dx.doi.org/10.21037/atm-21-2315).

\section{Methods}

\section{Patients and tissue samples}

CRC patients who received surgical resection at Jeonbuk National University Hospital from January 2018 to May 2019 , were enrolled in the study. For clinical stage IIII patients, radical resection was the first choice of treatment; for stage III patients, adjuvant chemotherapy (FOLFOX) was administered. Stage IV patients underwent palliative resection and received adjuvant chemotherapy. Clinicopathological characteristics were obtained from their medical records and are summarized in Table 1. The TNM classification of patients was based on the American Joint Committee on Cancer staging system $\left(8^{\text {th }}\right.$ edition). Assessment of tumor-infiltrating lymphocytes (TILs) was performed using the International TILs Working Group (ITWG) methodology (15). Serum tumor markers, including carcinoembryonic antigen (CEA) and cancer antigen 19-9 (CA19-9), were collected preoperatively, and their cut-off levels were $5.0 \mathrm{ng} / \mathrm{mL}$ and $37 \mathrm{kU} / \mathrm{L}$, respectively. The exclusion criterion was neoadjuvant chemotherapy or radiotherapy. For analysis, the entire colon was divided into the right colon and the left colon. The right colon was defined as the segment from the cecum to the proximal two-thirds of the transverse colon, and the left colon was defined as the segment from the distal onethird of the transverse colon to the rectum. Postoperative surveillance for CRC patients was performed every 3 months. Laboratory tests including serum tumor marker CEA and CA19-9 were performed. Abdominal computed tomography $(\mathrm{CT})$ was performed to detect recurrence and metastasis.

The study was conducted in accordance with the Declaration of Helsinki (as revised in 2013). The study was approved by the Institutional Review Board of Jeonbuk National University Hospital (IRB No. 2020-06-013-002), and individual consent for this retrospective analysis was waived.

\section{Next generation sequencing}

Targeted next-generation sequencing (NGS) was performed using formalin-fixed paraffin-embedded (FFPE) tumor tissue. Hematoxylin and eosin (H\&E)-stained slides were reviewed, and the tumor area with enough viable tumor 
Table 1 Association between patterns of PIK3CA mutation, PD-L1 expression and clinicopathological characteristics

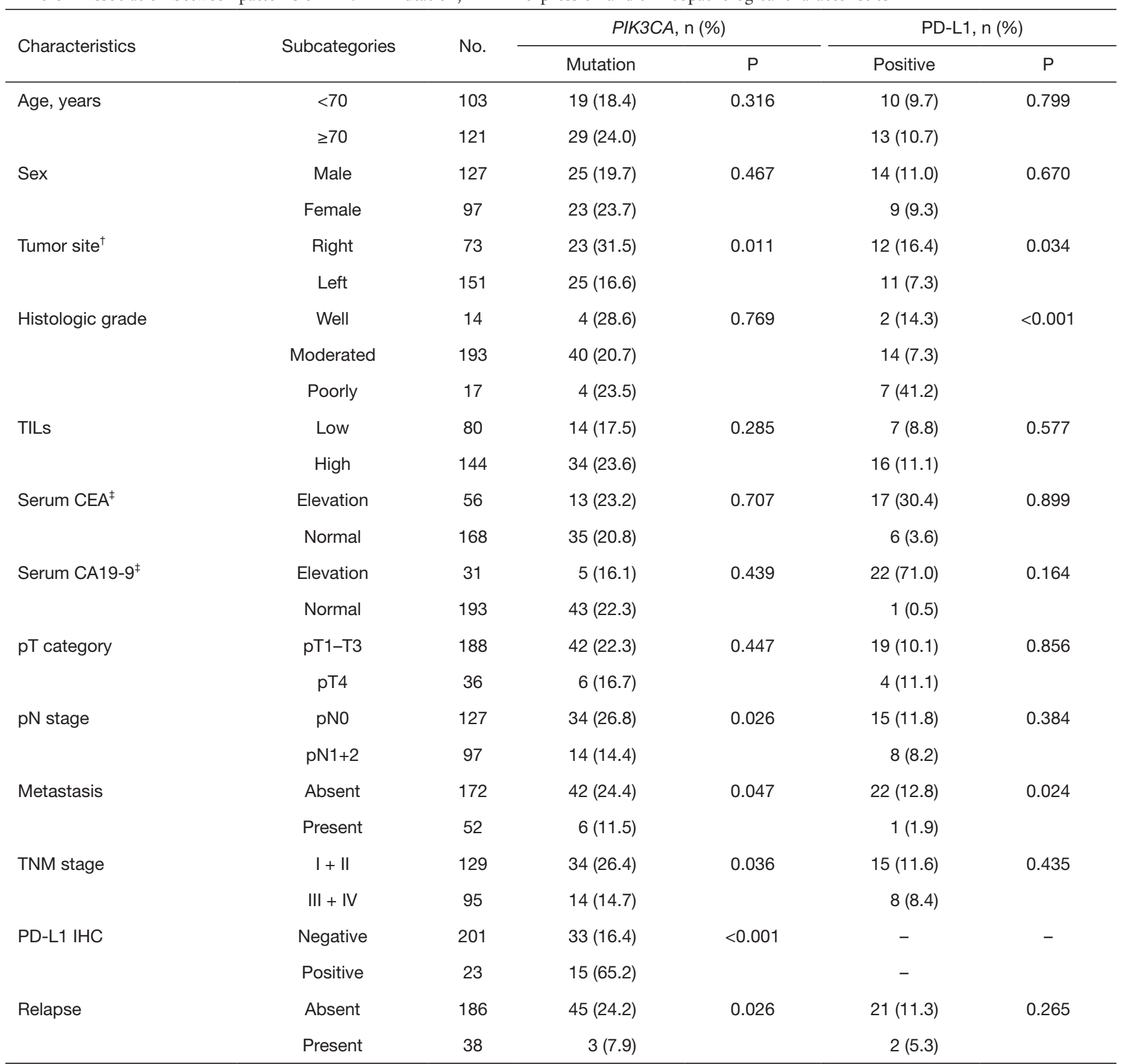

${ }^{\dagger}$, right colon: defined as the segment from the cecum to the proximal two-thirds of the transverse colon; left colon: defined as the segment from the distal one third of the transverse colon to the rectum. ${ }^{\ddagger}$, serum CEA elevation; defined as $>5.0 \mathrm{ng} / \mathrm{mL}$. Serum CA199 elevation defined as $>37$ kU/L. CA19-9, cancer antigen 19-9; CEA, carcinoembryonic antigen; IHC, immunohistochemistry; PD-L1, programmed death ligand-1; TILs, tumor infiltrating lymphocytes.

cells was marked and used as a guide for macrodissection. Areas with greater than $50 \%$ tumor volume were used for examination. In brief, total nucleic acid was isolated from FFPE tumor tissue using a RecoverAll Total Nucleic Acid Isolation Kit for FFPE (Ambion, Austin, TX, USA), according to the manufacturer's specifications. The samples were analyzed using the Oncomine Comprehensive Assay Cancer Panel (Ion Torrent S5 XL, Thermo Fisher Scientific, MA, USA) which covers 2,737 amplicons (2530 DNA + 207 RNA) within 143 cancer-related genes. 

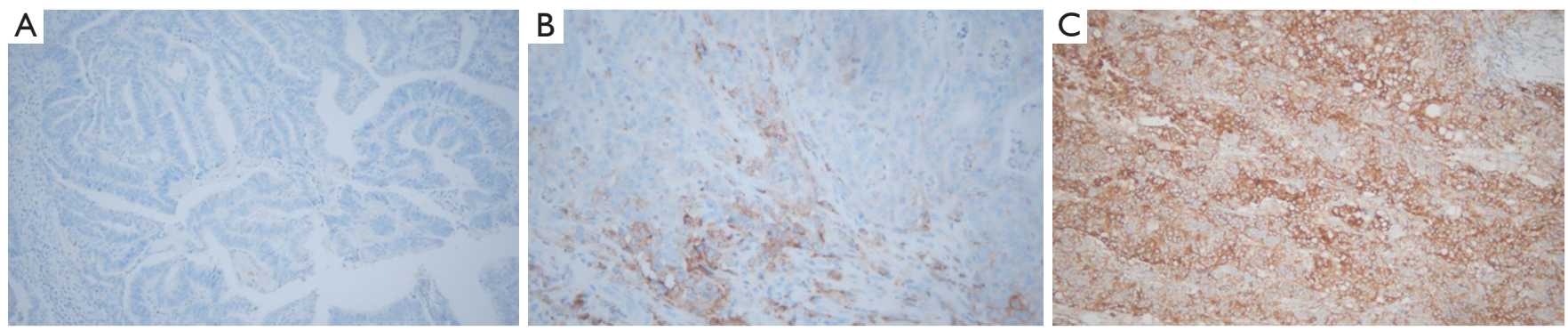

Figure 1 Expression of PD-L1 (SP263) in colorectal cancer. Only membranous staining of tumor cells was evaluated using immunohistochemical staining. (A) TPS $0:<1 \%$ of tumor cells; (B) TPS $1: \geq 1 \%$ to $49 \%$ of tumor cells; (C) TPS 2 : $\geq 50 \%$ of tumor cells. Magnification: 400×. PD-L1, programmed death ligand-1; TPS, tumor proportion score.

The percentage of amplicons covered was $95 \%$. Reads were aligned to the hg19 reference genome, and variants with allele frequencies less than $3 \%$ were excluded. The Oncomine Comprehensive Assay Cancer Panel included 15 amplicons located in PIK3CA exons 1, 2, 3, 4, 6, 7, 9, 13, 18, 19 , and 20.

\section{Evaluation of PD-L1 expression}

Immunohistochemical (IHC) staining was performed on tissue microarray (TMA) sections. FFPE tissue blocks containing representative samples of the tumors were selected by reviewing all H\&E-stained slides. The diameter of each TMA core was $4 \mathrm{~mm}$, and two cores per case were constructed. IHC staining with the primary antibody for PD-L1 (clone: SP263; dilution: ready-to-use; Roche) was performed using an automated immunostainer (BenchMark ULTRA, Roche Diagnostics, Mannheim, Germany) following the manufacturer's protocol. The slides stained for PD-L1 were evaluated by two pathologists (MJ Chung and AR Ahn) who had no clinicopathologic information on the patients. Only membranous staining of tumor cells was evaluated; nuclear and cytoplasmic staining was excluded from scoring (Figure 1). PD-L1 expression was defined as the percentage of tumor cells with membranous staining at any intensity. The percentage of PD-L1-positive tumor cells in the two TMA cores was averaged. The estimation of PDL1 expression was performed using the tumor proportion score (TPS), which is applied routinely in diagnostic settings (16). It is a three-point evaluation scale (0 points for $<1 \%, 1$ point for $\geq 1 \%$ to $49 \%$, and 2 points $\geq 50 \%$ ). The cut-off for positivity of PD-L1 was $\geq 1 \%$ of tumor cells of any intensity (17).

\section{Statistical analysis}

The association between molecular characteristics and clinicopathological factors was evaluated using the Chisquare test or Fisher's exact test. For each patient, the date of the last follow-up was that of the last contact or of death of patient up to April 2020. The median follow-up period was 16.1 months (range, 4-23 months). We evaluated the prognosis of CRC patients by analyzing overall survival (OS). Patient death from CRC was considered an event for OS analysis. Relapse-free survival (RFS) was defined as the time from primary surgical treatment to detection of relapse or death. Survival curves were plotted using the Kaplan-Meier method and analyzed using the log-rank test. We performed univariate and multivariate Cox proportional hazards regression analyses, Kaplan-Meier survival analysis, and Pearson's Chi-square tests using SPSS software version 19.0 (IBM Corp., Armonk, NY, USA), with $\mathrm{P}<0.05$ indicating a significant difference.

\section{Results}

\section{Genetic alterations of PIK3CA and their correlation with clinicopathological parameters in CRC patients}

A total of 224 patients with primary CRC was enrolled in this study. The mean age was 69.2 years (range, 3591 years). Overall, $127(56.7 \%)$ patients were male and 97 (43.3\%) were female (Table 1). Analysis of PIK3CA mutations was performed using targeted NGS, which examined 160 hotspots and included single-nucleotide variants and deletions. PIK3CA mutations were observed in $21.4 \%(48 / 224)$ of the patients. A total of 27 cases (56.3\%) of PIK3CA mutations was detected in exon 9, and 12 cases $(25.0 \%)$ in exon 20 . In two cases, two types of PIK3CA 
Table 2 Association between site of PIK3CA mutation and PD-L1 expression

\begin{tabular}{lccc}
\hline \multirow{2}{*}{ PIK3CA mutation } & No. & \multicolumn{2}{c}{ PD-L1 } \\
\cline { 3 - 4 } & & Positive, n (\%) & P \\
\hline Exon & 27 & $11(40.7)$ & $<0.001$ \\
9 & 12 & $3(25.0)$ & \\
20 & 11 & $3(27.3)$ & $<0.001$ \\
Other & 176 & $8(4.5)$ & \\
Wild type & & & \\
Domain & 20 & $9(45.0)$ & \\
Helical D & 9 & $1(11.1)$ & \\
Kinase D & 21 & $7(33.3)$ & \\
Other & 176 & $8(4.5)$ & \\
Wild type & & & \\
\hline
\end{tabular}

PD-L1, programmed death ligand-1.

mutations were detected. Of all PIK3CA mutations, 56.3\% were identified in three major hotspots: E545K $(22.9 \%)$ and $\mathrm{E} 542 \mathrm{~K}(16.7 \%)$ in exon 9 and H1047R/L (16.7\%) in exon 20 (Table S1). PIK3CA mutations were more significantly associated with cancer in the right colon than in the left colon $(\mathrm{P}=0.011)$. PIK3CA mutations were inversely correlated with lymph node metastasis $(\mathrm{P}=0.026)$, distant metastasis $(\mathrm{P}=0.047)$, and high TNM stage $(\mathrm{P}=0.036)$ (Table 1).

\section{Expression of PD-L1 and its correlation with PIK3CA mutations in CRC patients}

PD-L1 expression was found in 23 of 224 cases (10.3\%): 19 cases with TPS 1 and 4 cases with TPS 2. The results revealed that $\mathrm{PD}-\mathrm{L} 1$ expression was more significantly associated with right-sided colon cancer $(\mathrm{P}=0.034)$ and poorly differentiated carcinoma $(\mathrm{P}<0.001)$. PD-L1 expression was correlated inversely with distant metastasis $(\mathrm{P}=0.024)$ (Table 1).

Additionally, PD-L 1 expression was associated significantly with $P I K 3 C A$ mutations $(\mathrm{P}<0.001)$. In particular, the exon 9 (helical domain) mutations of $P I K 3 C A$ were significantly associated with PD-L1 expression $(\mathrm{P}<0.001)$ (Table 2). However, there was no relationship between the TPS level of PD-L1 expression and PIK3CA mutations. PD-L1 expression was found in 15 of $48(31.3 \%)$ cases of PIK3CA mutation: 12 cases with TPS 1 and three cases with TPS 2. In PIK3CA non-mutated cases, $4.5 \%$ (8/176) showed PD-L1 expression: seven cases with TPS 1 and one case with TPS 2.

\section{Relationship between PIK3CA mutation and the prognosis of patients with CRC}

In univariate survival analysis, the factors significantly associated with OS were poor histologic differentiation $(\mathrm{P}<0.001)$, elevated preoperative serum level of CA19-9 $(\mathrm{P}<0.001)$ and CEA $(\mathrm{P}=0.015)$, high $\mathrm{T}$ category $(\mathrm{P}<0.001)$, lymph node metastasis $(\mathrm{P}=0.019)$, presence of distant metastasis $(\mathrm{P}<0.001)$, and high tumor stage $(\mathrm{P}=0.014)$. The PIK3CA mutation were not correlated with OS. Low TILs $(\mathrm{P}=0.025)$, CA19-9 elevation $(\mathrm{P}<0.001)$, high $\mathrm{T}$ category $(\mathrm{P}<0.001)$, lymph node metastasis $(\mathrm{P}<0.001)$, presence of distant metastasis $(\mathrm{P}<0.001)$, high tumor stage $(\mathrm{P}<0.001)$, and wild $P I K 3 C A(\mathrm{P}=0.048)$ were significantly associated with shorter RFS (Table 3). Patients with a PIK3CA mutation exhibited better RFS compared to that of the wild-type $P I K 3 C A$ group. To confirm that the correlation between the PIK3CA mutations and longer RFS was not caused by sampling bias, the effect of PIK3CA mutation on RFS was examined only in CRC patients with a low $\mathrm{T}$ category. The CRC patients with a low $\mathrm{T}$ category who had PIK3CA mutations exhibited longer RFS compared to those with wild-type PIK3CA ( $\mathrm{P}=0.028)$ (Figure 2). The factors that had significant or borderline significant $(\mathrm{P}<0.1)$ correlation with OS or RFS by univariate analysis were included in the multivariate analysis. In multivariate analysis, CA19-9 elevation $(\mathrm{P}=0.005)$ and $\mathrm{T}$ category $(\mathrm{P}=0.045)$ were independent indicators for OS of patients with CRC. Distant metastasis was an independent indicator of RFS $(\mathrm{P}<0.001)$. No variables were significantly correlated with both OS and RFS (Table 4).

\section{Discussion}

In this study, we investigated PIK3CA mutations and IHC expression of PD-L1 in human CRC tissues. Our results show: (I) PIK3CA mutations in $21.4 \%$ of CRC (48/224) samples; (II) PIK3CA mutations correlated with negative lymph node status, low tumor stage, and absence of distant metastasis; (III) PIK3CA mutations correlated with longer RFS by univariate analysis; and (IV) PIK3CA mutations correlated with PD-L1 expression.

The mutation of PIK3CA is the most common alteration in the PI3K-AKT pathway and plays an important role in 
Table 3 Univariate Cox proportional hazards regression analysis for the overall survival and relapse-free survival of CRC patients

\begin{tabular}{|c|c|c|c|c|c|}
\hline Characteristics & No. & \multicolumn{2}{|l|}{ OS } & \multicolumn{2}{|l|}{ RFS } \\
\hline Age, years, $\geq 70$ (vs. <70) & $121 / 224$ & $1.935(0.840-4.454)$ & 0.121 & $1.680(0.858-3.291)$ & 0.130 \\
\hline Sex, female (vs. male) & $97 / 224$ & $0.734(0.327-1.650)$ & 0.455 & $1.170(0.616-2.222)$ & 0.632 \\
\hline Tumor site, left colon (vs. right colon) & $151 / 224$ & $0.647(0.297-1.410)$ & 0.274 & $0.794(0.411-1.536)$ & 0.493 \\
\hline High TIL (inter + high) (vs. low) & $144 / 224$ & $0.677(0.312-1.466)$ & 0.322 & $0.427(0.225-0.809)$ & 0.009 \\
\hline CEA elevation (vs. normal range) & $56 / 224$ & $2.648(1.209-5.800)$ & 0.015 & $1.815(0.907-3.632)$ & 0.092 \\
\hline CA19-9 elevation (vs. normal range & $31 / 224$ & $6.890(3.164-15.006)$ & $<0.001$ & $4.860(2.456-9.616)$ & $<0.001$ \\
\hline pT category, pT4 (vs. pT1-pT3) & $36 / 224$ & $4.501(2.063-9.818)$ & $<0.001$ & $4.331(2.230-8.413)$ & $<0.001$ \\
\hline Stage, $\geq$ III \& IV (vs. I \& II) & $95 / 224$ & $2.758(1.229-6.190)$ & 0.014 & 4.025 (1.994-8.124) & $<0.001$ \\
\hline PD-L1, positive (vs. negative & $23 / 224$ & $0.955(0.310-3.453)$ & 0.955 & $0.391(0.094-1.629)$ & 0.197 \\
\hline PIK3CA, mutated (vs. wild-type) & $48 / 224$ & $1.402(0.589-3.337)$ & 0.446 & $0.304(0.093-0.988)$ & 0.048 \\
\hline
\end{tabular}

CA19-9, cancer antigen 19-9; CEA, carcinoembryonic antigen; Cl, confidence interval; CRC, colorectal cancer; HR, hazard ratio; OS, overall survival; PD-L1, programmed death ligand-1; RFS, relapse-free survival; TILs, tumor infiltrating lymphocytes.
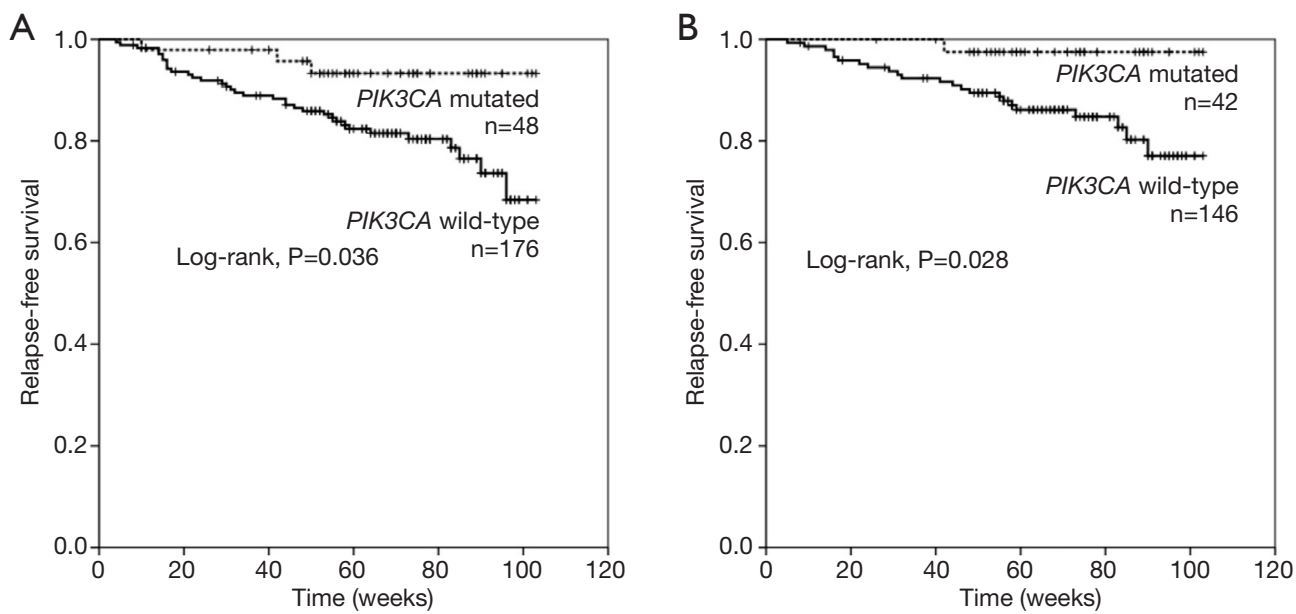

Figure 2 A Kaplan-Meier curve of relapse-free survival according to PIK3CA status. (A) Mutation of PIK3CA in 224 CRC patients was significantly associated with relapse-free survival; (B) in patients with low T category CRC, PIK3CA mutations were significantly associated with relapse-free survival. CRC, colorectal cancer.

the pathogenesis of CRC (18). Previous studies report that $14-32 \%$ of patients with CRC have PIK3CA mutations (19). Somatic missense mutations of PIK3CA were scattered across most of the exons but were found predominantly in the kinase (H1047R) and helical (E542K and E545K) domains of the PIK3CA subunit (20). Our results are similar to those of previous studies. PIK3CA mutations were observed in $48(21.4 \%)$ of the 224 CRC samples, and the most frequently recurring mutations were E545K (22.9\%), E542K (16.7\%), and H1047R/L (16.7\%). 
Table 4 Multivariate Cox regression analysis for the overall survival and relapse-free survival of CRC patients

\begin{tabular}{lcccc}
\hline \multirow{2}{*}{ Characteristics } & \multicolumn{2}{c}{$\mathrm{OS}^{\dagger}$} & \multicolumn{2}{c}{$\mathrm{RFS}^{\ddagger}$} \\
\cline { 2 - 3 } CA19-9 elevation (vs. normal range) & $\mathrm{HR}(95 \% \mathrm{Cl})$ & $\mathrm{P}$ & $\mathrm{HR}(95 \% \mathrm{Cl})$ \\
PT category, pT4 (vs. pT1-pT3) & $3.853(1.514-9.809)$ & 0.005 & $1.459(0.677-3.144)$ & 0.335 \\
Distant metastasis, presence (vs. absence) & $2.492(1.021-6.080)$ & 0.045 & $1.391(0.672-2.878)$ & 0.374 \\
\hline
\end{tabular}

${ }^{\dagger}$, variables considered in multivariate analysis for overall survival were CEA elevation, CA19-9 elevation, T stage, lymph node metastasis, distant metastasis, stage. ${ }^{\ddagger}$, variables considered in multivariate analysis for relapse-free survival were CEA elevation, CA19-9 elevation, TILs, T stage, lymph node metastasis, distant metastasis, stage, and PIK3CA mutation. CA19-9, cancer antigen 19-9; CI, confidence interval; CRC, colorectal cancer; HR, hazard ratio; OS, overall survival; RFS, relapse-free survival.

PIK3CA mutations and prognosis in human cancers have yielded variable results $(10,21,22)$. Previous studies have indicated that PIK3CA mutations are oncogenic and associated with an aggressive CRC phenotype and/ or poor prognosis $(16,23,24)$. It has been suggested that PIK3CA mutations either increase kinase activity or cause overexpression of these mutant PIK3CA proteins, which leads to concomitant phosphorylation of proteins in the AKT pathway. The activated AKT pathway plays an important role in tumor proliferation, survival, invasion, and angiogenesis, through which it exhibits aggressive tumor behavior. However, some studies have reported different results. PIK3CA mutations have been associated with a favorable cancer phenotype, and recent reports using metaanalysis have found no difference in PIK3CA mutations with relation to prognosis (10). Therefore, there is controversy surrounding the clinical significance of PIK3CA mutations. Our study revealed that PIK3CA mutations were correlated with favorable clinicopathologic factors and longer RFS in CRC patients.

The relationship between the PIK3CA mutations and good prognosis can be explained as follows. First, to obtain malignant properties, AKT inactive tumors (i.e., tumors in which the PI3K-AKT pathway is not activated) must have an abnormality that can be an alternative to AKT activation. These deviations can lead to more aggressive behavior than that with AKT activation (11). Second, in a study examining the clinical significance of PIK3CA overexpression in ovarian clear cell carcinoma, carcinomas containing activated PIK3CA had a better prognosis compared to tumors without activated PIK3CA. It has been asserted that the observed effect of PIK $3 C A$ on prognosis was not due to the function of downstream effectors but to PIK3CA genetic modification (25). A third possible explanation for the relationship between
PIK3CA mutations and favorable prognosis is "oncogeneinduced senescence", an endogenous tumor suppression mediated by replication stress (26). Oncogenes are key factors in carcinogenesis that trigger activation of various signaling pathways and downstream effectors. However, oncogenes also act as tumor suppressors by activating senescence. Activation of the PI3K-AKT pathway by the PIK3CA mutations lead to accumulation of reactive oxygen species (ROS) that induce activation of the p53/p21WAF1 pathway and senescence (27). ROS are major factors that induce cell cycle arrest and cellular senescence (28). Unfortunately, there is no satisfactory explanation for these conflicting results. Elucidating how mutations in PIK3CA achieve this opposite effect (favorable versus poor prognosis) will be important in understanding the pathogenesis of CRC. In addition, this knowledge could be used to determine therapeutic approaches for patients with PIK3CA mutations.

Our results show PIK3CA mutations correlated with favorable prognostic factors and longer RFS in the univariate analysis, but this was not supported by the multivariate analysis. Additionally, right-sided colon cancer had a higher rate of PIK3CA mutations, and these results are consistent with previous studies. The outcomes of patients with left-sided cancers were better than those with rightsided cancers in instances of metastatic CRC. In relation to prognosis, the contradiction shown by tumor sidedness and PIK3CA mutations needs to be confirmed through additional studies. This result is presumed to be affected by the short follow up time ( 23 months), a limitation of this study. Further studies are required to confirm our results.

The standard treatment for advanced CRC is surgical resection and adjuvant chemotherapy. Conventional therapy has a limited therapeutic effect in a subset of patients with CRC (2). Immunotherapy for cancer patients has several 
advantages, such as high accuracy and fewer side effects compared to conventional adjuvant chemotherapy (29). There are several immune checkpoint inhibitors (ICIs) approved by the Food and Drug Administration that target different pathways, including PD-1, PD-L1, and the cytotoxic $\mathrm{T}$ lymphocyte antigen 4 pathway. PD-L1 expression is a well-known predictive biomarker for PD-1/ PD-L1 inhibitors in advanced cancer patients. The PI3K signaling pathway is involved in the immune response of tumors, including the anti-cancer immune response, and the acquisition of leukocyte-like properties by cancer cells (30). Studies have shown that PD-L1 expression is regulated by transcription factors, signaling pathways, and epigenetic factors. The PI3K/AKT signaling pathway has been shown to affect tumoral PD-L1 expression. However, the association between PIK3CA mutation and PD-L1 expression in human CRC has not been established. Nusrat et al. have shown that $\mathrm{PD}-\mathrm{L} 1$ expression is significantly correlated with PIK3CA mutations (31). Interaction between the helical domain mutation of PIK3CA and Ras-GTP can increase PD-L1 mRNA stability, which increases PD-L1 surface expression (32). Others have found no correlation between PIK3CA and PD-L1 (27). In the current study, PD-L1 expression was found in 23 of 224 cases (10.3\%) and was significantly associated with PIK3CA mutations $(\mathrm{P}<0.001)$. In particular, the exon 9 (helical domain) mutations of PIK3CA were associated significantly with PD-L1 expression $(\mathrm{P}<0.001)$. A previous study has shown that PIK3CA mutations are associated with greater clinical benefit from immunotherapy (30). Based on these results, it would be meaningful to further investigate the mechanism of the relationship between the PIK3CA mutation and PD-L1 expression. Future research into the mechanism is expected to assist in selection of more effective candidates for immunotherapy.

In the current study, we investigated PIK3CA mutations in $\mathrm{CRC}$ and the relationship between PIK3CA mutations and patient clinicopathological characteristics as well as $\mathrm{PD}$ L1 expression. PIK3CA mutations were observed in $21.4 \%$ of CRCs and were associated with favorable prognostic factors and longer RFS. We discussed possible mechanisms that can explain the association between PIK3CA mutations and favorable prognosis in cancer. In addition, we showed that PIK3CA mutations had a significant correlation with expression of PD-L1, which suggests that cancer with both PIK3CA mutations and PD-L1 expression is a good candidate for immunotherapy. Further investigation is required to identify whether PIK3CA mutations are a good prognostic factor. In addition, further studies are needed to understand the mechanisms of the association between PIK3CA mutations and favorable prognosis of cancer and the overall correlation between PIK3CA mutations and PDL1 expression.

\section{Acknowledgments}

Funding: This paper was supported by Fund of Biomedical Research Institute, Chonbuk National University Hospital.

\section{Footnote}

Reporting Checklist: The authors have completed the REMARK reporting checklist. Available at https://dx.doi. org/10.21037/atm-21-2315

Data Sharing Statement: Available at https://dx.doi. org/10.21037/atm-21-2315

Peer Review File: Available at https://dx.doi.org/10.21037/ atm-21-2315

Conflicts of Interest: All authors have completed the ICMJE uniform disclosure form (available at https://dx.doi. org/10.21037/atm-21-2315). The authors have no conflicts of interest to declare.

Ethical Statement: The authors are accountable for all aspects of the work in ensuring that questions related to the accuracy or integrity of any part of the work are appropriately investigated and resolved. The study was conducted in accordance with the Declaration of Helsinki (as revised in 2013). The study was approved by the Institutional Review Board of Jeonbuk National University Hospital (IRB No. 2020-06-013-002) and individual consent for this retrospective analysis was waived.

Open Access Statement: This is an Open Access article distributed in accordance with the Creative Commons Attribution-NonCommercial-NoDerivs 4.0 International License (CC BY-NC-ND 4.0), which permits the noncommercial replication and distribution of the article with the strict proviso that no changes or edits are made and the original work is properly cited (including links to both the formal publication through the relevant DOI and the license). See: https://creativecommons.org/licenses/by-nc-nd/4.0/. 


\section{References}

1. Bray F, Ferlay J, Soerjomataram I, et al. Global cancer statistics 2018: GLOBOCAN estimates of incidence and mortality worldwide for 36 cancers in 185 countries. CA Cancer J Clin 2018;68:394-424.

2. Malet-Martino M, Martino R. Clinical studies of three oral prodrugs of 5-fluorouracil (capecitabine, UFT, S-1): a review. Oncologist 2002;7:288-323.

3. Shaw RJ, Cantley LC. Ras, PI(3)K and mTOR signalling controls tumour cell growth. Nature 2006;441:424-30.

4. Halilovic E, She QB, Ye Q, et al. PIK3CA mutation uncouples tumor growth and cyclin D1 regulation from MEK/ERK and mutant KRAS signaling. Cancer Res 2010;70:6804-14.

5. Trejo CL, Green S, Marsh V, et al. Mutationally activated PIK3CA(H1047R) cooperates with BRAF(V600E) to promote lung cancer progression. Cancer Res 2013;73:6448-61.

6. Sartore-Bianchi A, Martini M, Molinari F, et al. PIK3CA mutations in colorectal cancer are associated with clinical resistance to EGFR-targeted monoclonal antibodies. Cancer Res 2009;69:1851-7.

7. Mei ZB, Duan CY, Li CB, et al. Prognostic role of tumor PIK3CA mutation in colorectal cancer: a systematic review and meta-analysis. Ann Oncol 2016;27:1836-48.

8. Baba Y, Nosho K, Shima K, et al. Phosphorylated AKT expression is associated with PIK3CA mutation, low stage, and favorable outcome in 717 colorectal cancers. Cancer 2011;117:1399-408.

9. Christensen TD, Palshof JA, Larsen FO, et al. Associations between primary tumor RAS, BRAF and PIK3CA mutation status and metastatic site in patients with chemo-resistant metastatic colorectal cancer. Acta Oncol 2018;57:1057-62.

10. Alqahtani A, Ayesh HSK, Halawani H. PIK3CA Gene Mutations in Solid Malignancies: Association with Clinicopathological Parameters and Prognosis. Cancers (Basel) 2019;12:93.

11. Liao X, Morikawa T, Lochhead P, et al. Prognostic role of PIK3CA mutation in colorectal cancer: cohort study and literature review. Clin Cancer Res 2012;18:2257-68.

12. Gu Z. cBioPortal. 2016. Available online: http://www. cbioportal.org

13. Voutsadakis IA. The Landscape of PIK3CA Mutations in Colorectal Cancer. Clin Colorectal Cancer 2021;20:201-15.

14. Menyhárt O, Pongor LS, Győrffy B. Mutations Defining Patient Cohorts With Elevated PD-L1 Expression in
Gastric Cancer. Front Pharmacol 2018;9:1522.

15. Fuchs TL, Sioson L, Sheen A, et al. Assessment of Tumorinfiltrating Lymphocytes Using International TILs Working Group (ITWG) System Is a Strong Predictor of Overall Survival in Colorectal Carcinoma: A Study of 1034 Patients. Am J Surg Pathol 2020;44:536-44.

16. Ogino S, Nosho K, Kirkner GJ, et al. PIK3CA mutation is associated with poor prognosis among patients with curatively resected colon cancer. J Clin Oncol 2009;27:1477-84.

17. Kintsler S, Cassataro MA, Drosch M, et al. Expression of programmed death ligand (PD-L1) in different tumors. Comparison of several current available antibody clones and antibody profiling. Ann Diagn Pathol 2019;41:24-37.

18. Mármol I, Sánchez-de-Diego C, Pradilla Dieste A, et al. Colorectal Carcinoma: A General Overview and Future Perspectives in Colorectal Cancer. Int J Mol Sci 2017;18:197.

19. Cohen SA, Turner EH, Beightol MB, et al. Frequent PIK3CA Mutations in Colorectal and Endometrial Tumors With 2 or More Somatic Mutations in Mismatch Repair Genes. Gastroenterology 2016;151:440-447.e1.

20. Abubaker J, Bavi P, Al-Harbi S, et al. Clinicopathological analysis of colorectal cancers with PIK3CA mutations in Middle Eastern population. Oncogene 2008;27:3539-45.

21. Pergialiotis V, Nikolaou C, Haidopoulos D, et al. PIK3CA Mutations and Their Impact on Survival Outcomes of Patients with Cervical Cancer: A Systematic Review. Acta Cytol 2020;64:547-55.

22. Jin J, Shi Y, Zhang S, et al. PIK3CA mutation and clinicopathological features of colorectal cancer: a systematic review and Meta-Analysis. Acta Oncol 2020;59:66-74.

23. Guo XN, Rajput A, Rose R, et al. Mutant PIK3CA-bearing colon cancer cells display increased metastasis in an orthotopic model. Cancer Res 2007;67:5851-8.

24. Kato S, Iida S, Higuchi T, et al. PIK3CA mutation is predictive of poor survival in patients with colorectal cancer. Int J Cancer 2007;121:1771-8.

25. Abe A, Minaguchi T, Ochi H, et al. PIK3CA overexpression is a possible prognostic factor for favorable survival in ovarian clear cell carcinoma. Hum Pathol 2013;44:199-207.

26. Halazonetis TD, Gorgoulis VG, Bartek J. An oncogeneinduced DNA damage model for cancer development. Science 2008;319:1352-5.

27. Albitar M, Sudarsanam S, Ma W, et al. Expression of PD-L1 in colorectal cancer that lack mutations in 


\section{Page 10 of 10}

RAS or TP53 genes. J Clin Oncol 2017. doi: 10.1200/ JCO.2017.35.15_suppl.e14500

28. Davalli P, Mitic T, Caporali A, et al. ROS, Cell Senescence, and Novel Molecular Mechanisms in Aging and Age-Related Diseases. Oxid Med Cell Longev 2016;2016:3565127.

29. Burger JA, Tedeschi A, Barr PM, et al. Ibrutinib as Initial Therapy for Patients with Chronic Lymphocytic Leukemia. N Engl J Med 2015;373:2425-37.

30. Dituri F, Mazzocca A, Giannelli G, et al. PI3K functions

\section{Ahn et al. PIK3CA mutation and PD-L1 expression in CRC}

in cancer progression, anticancer immunity and immune evasion by tumors. Clin Dev Immunol 2011;2011:947858.

31. Nusrat M, Roszik J, Katkhuda R, et al. Association of PIK3CA mutations (mut) with immune engagement and clinical benefit from immunotherapy in microsatellite stable (MSS) colorectal cancer (CRC) patients (pts). J Clin Oncol 2019;37:abstr 3604.

32. Glodde N, Hölzel M. RAS and PD-L1: A Masters' Liaison in Cancer Immune Evasion. Immunity 2017;47:1007-9.

Cite this article as: Ahn AR, Kim KM, Jang KY, Moon WS, Ha GW, Lee MR, Chung MJ. Correlation of PIK3CA mutation with programmed death ligand-1 (PD-L1) expression and their clinicopathological significance in colorectal cancer. Ann Transl Med 2021;9(18):1406. doi: 10.21037/atm-21-2315 


\section{Supplementary}

Table S1 Summary of PIK3CA mutation in colorectal cancers

\begin{tabular}{|c|c|c|c|c|}
\hline Exon & Nucleotide & AA mutation & Domain & Cases \\
\hline 9 & c. $1633 G>A$ & p.E545K & Helical & 11 \\
\hline 9 & c. $1624 G>A$ & p.E542K & Helical & 8 \\
\hline 9 & c. $1636 C>G$ & p.Q546E & Helical & 3 \\
\hline 9 & c. $1637 A>G$ & p.Q546R & Helical & 3 \\
\hline 9 & c. $1635 \mathrm{G}>\mathrm{C}$ & p.E545D & Helical & 1 \\
\hline 9 & c. $1637 A>C$ & p.Q546P & Helical & 1 \\
\hline 20 & c. $3140 A>G$ & p.H1047R & Kinase & 6 \\
\hline 20 & c. $3140 \mathrm{~A}>\mathrm{T}$ & p.H1047L & Kinase & 2 \\
\hline 20 & c. $3139 \mathrm{C}>\mathrm{T}$ & p.H1047Y & Kinase & 1 \\
\hline 20 & c. $3061 \mathrm{~T}>\mathrm{C}$ & p.Y1021H & Kinase & 1 \\
\hline 20 & c. $.3085 \mathrm{G}>\mathrm{T}$ & p.D1029Y & Kinase & 1 \\
\hline 20 & c. $3129 \mathrm{G}>\mathrm{A}$ & p.M1043I & Kinase & 1 \\
\hline 4 & c. $1031 \mathrm{~T}>\mathrm{G}$ & p.V344G & C2 & 2 \\
\hline 4 & c. $1049 A>G$ & p.D350G & C2 & 1 \\
\hline 4 & c. $1030 \mathrm{G}>\mathrm{A}$ & p.V344M & C2 & 1 \\
\hline 1 & c. $277 \mathrm{C}>\mathrm{T}$ & p.R93W & $A B D$ & 1 \\
\hline 1 & c. $263 \mathrm{G}>\mathrm{A}$ & p.R88Q & $A B D$ & 1 \\
\hline 1 & c. $112 \mathrm{C}>\mathrm{T}$ & p.R38C & $A B D$ & 1 \\
\hline 1 & c.328_330delGAA & p.E109del & $\mathrm{N} / \mathrm{S}$ & 1 \\
\hline 7 & c. $1258 \mathrm{~T}>\mathrm{C}$ & p.C420R & C2 & 1 \\
\hline 13 & c. $2176 \mathrm{G}>\mathrm{A}$ & p.E726K & $\mathrm{N} / \mathrm{S}$ & 1 \\
\hline 5 & c. $1090 \mathrm{G}>\mathrm{A}$ & p.G364R & C2 & 1 \\
\hline
\end{tabular}

$A A$, amino acid; $A B D$, adapter binding domain; N/S, not specified. 